\title{
Comment on: The value of post-operative MR in tethered cord: a review of 140 cases by P. David Halevi, Suhas Udayakumaran, Liat Ben-Sira, Shlomi Constantini
}

\author{
Paul Steinbok
}

Received: 4 September 2011 /Accepted: 4 September 2011 / Published online: 29 September 2011

(C) Springer-Verlag 2011

Halevi et al. have reviewed the value of routine postoperative MRI after cord untethering procedures and have concluded that "postoperative MR as routine clinical practice is not justified for uncomplicated cases of tethered cord release." I agree with these conclusions, and indeed, in my own clinical practice, I have not been doing routine postoperative MR scans after simple untethering procedures.

Often, surgeons request postoperative MR scans to see what they have achieved at surgery and to obtain a baseline with which to compare future studies. Knowing what has been achieved at surgery may be important in many situations, such as tumor resection. However, in surgery for cord untethering, this indication for postoperative MR scanning may be valid only when there was a preexisting syrinx or after more complicated procedures such as resection of a dermoid or conus lipoma. Certainly, in a straightforward cord untethering procedure, doing a postoperative scan to see what was achieved at surgery is not required.

As for the second indication for postoperative MR scanning, namely to obtain a baseline scan, this is important only if future management will be based in part on identifying changes in future scans when compared to the "baseline" scan done postoperatively. In cord untethering procedures, such a baseline scan may be important in following a known syrinx or dermoid cyst and in identifying the rare cases where residual lipoma grows and is a cause of new symptoms and signs. However, for those cases where there was a simple cord untethering, with section of the filum terminale or lysis of tethering bands, consideration of further surgery is based on the development of new clinical findings. If that should occur, a repeated MR scan will be done to rule out a new problem that might be responsible for the new clinical findings. In the absence of any new radiological finding, a presumptive diagnosis of symptomatic retethering will be considered and management will proceed accordingly. Comparison with a prior postoperative "baseline" scan will not change the diagnosis or management in these situations. Hence, the postoperative baseline scan is of no value in the management and is not indicated routinely.

MR scans are costly, and in many centers, MR scanning is a scarce resource with long waits for a nonurgent scan. Furthermore, in young children, sedation and even general anesthesia are often required to get a high-quality MR scan. It is important to request MR scans and, indeed, any investigation only when the information provided will be useful in the management of the patient.

Although the study of Halevi et al. will not change my practice, since I do not order routine postoperative MR scans after simple cord untethering procedures, I congratulate the authors in reporting their findings and challenging us to think more about why we order such investigations.

P. Steinbok $(\bowtie)$

British Columbia Children's Hospital,

Vancouver, BC, Canada

e-mail: psteinbok@cw.bc.ca 